\title{
Research on the Strategy How to Clean up Space Debris \\ Bin Yang
}

School of Electric Power Engineering, North China Electric Power University, Baoding 071000, China.

1422714487@qq.com

Keywords: space debris, Simulated Annealing, Double Weibull probability distribution, Objective optimization model

\begin{abstract}
Now space debris poses a serious threat to humanities efforts at space exploration as well as the expanding uses for earth-orbiting satellites. This thesis is to determine the best alternative for a private firm to address the space debris problem. To determine the best alternative or combination of alternatives that a private firm could adopt as a commercial opportunity to address the space debris problem, we have to determine which space debris are proposed to clean. First, we construct the Space Debris Growth Model to predict the growth trend of space debris by using Least Squares and Simulated Annealing based on statistics in past years. Then, the Orbital Distribution Model of Space Debris are built to gain the space debris distribution and we simulate the space debris distribution by Double Weibull probability distribution model to find the probability of debris in diffident altitude. Next, by the aforementioned time-dependent model, we define several parameters $D_{\text {sum }}, \operatorname{Dan}_{h}, f$ to measure the cost, risks, benefits of space debris in different situations. Finally, concerned about these factors, we built objective function, which are solved by using Simulated Annealing. In this way, we obtain the best clean-up strategy, consisted of the time when to clean, the debris which to remove and the method which to choose.
\end{abstract}

\section{Introduction}

Since 1957, the Soviet Union launched Sputnik satellite, over the last few decades, the rate at which human activities in space have led to the creation of space debris has increased in a linear fashion. As the film <Gravity> presents a huge disaster caused by space debris, many space debris have posed a great threat to human space resource development activities. For example, the Russian satellite Kosmos-2251 and the USA satellite Iridium-33 collided on 10 February, 2009, which has a great impact on space environment. So we have to try our best to control and slow down the space debris.

\section{Assumptions}

As to simplify the problem, we make the following assumptions in the process of building model in this paper:

- The growth of space debris depends on known sources and we do not consider unknown sources.

- Space debris is independent with each other.

- In terms of space debris' risk to the satellite, we only considers the operational risk, we do not consider the risk of satellite launch.

- The quantity and cost of cleaning equipment is replaced by the removing cost in optimization model. 
Table1 symbol for basic model

\begin{tabular}{cl}
\hline $\begin{array}{c}\text { Symbol } \\
Q_{i}\end{array}$ & Definition \\
\hline$f(h)$ & The amount of space debris numbered $i$ \\
The PDF of the Space Debris Amount & The CDF of the Space Debris Amount \\
\hline Symbol & Table2 symbol for advanced model \\
\hline$f$ & The risk of a single space debris \\
$\sigma_{i}$ & The parameter to present whether the debris is to remove \\
$\omega_{i}$ & The weight of the cost to remove different debris \\
$\Delta M$ & The quality of the debris increased in one year \\
$\Delta F$ & The risk of the total debris increased in one year
\end{tabular}

\section{Basic model}

\section{The growth of space debris}

To determine which debris are supposed to clean, we should know the growth of space debris with time going by. Using the historical data from website ${ }^{[]}$, we can get the general trend of change of the debris' quantity in different size and the results are shown as Figure 1. Based on the diagram Figure 1, we obtain the growth trend of various types of space debris with time going by, by applying the method of fitting to calculate with MATLAB and the results are shown as Figure 2.
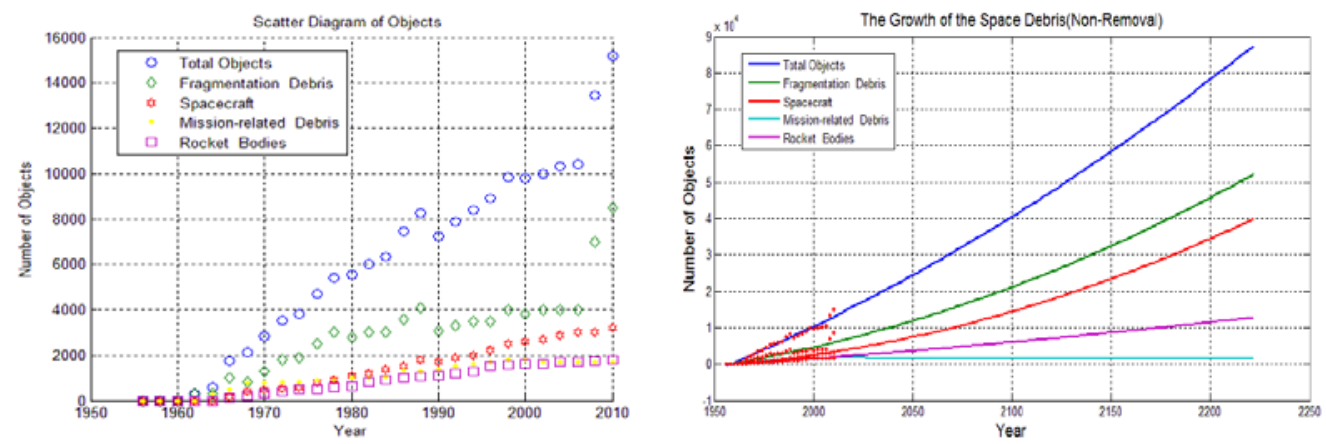

Figure 1.Scatter Diagram of Objects Figure 2. The Growth of the Space Debris

\section{The Orbital Distribution of space debris}

Based on the literature, we learn that the distribution of orbit debris is defined as the spatial density, which represents the number of debris existing in unite volume, and its size stand for the probability of collisions between spacecraft and the debris. So we are supposed to study the Orbital Distribution of space debris.

We extract the altitude and the number of space debris data from the data on website. Then we discard the data with limit value and draw the frequency distribution of space debris against altitude. The certain result is shown as follows: 


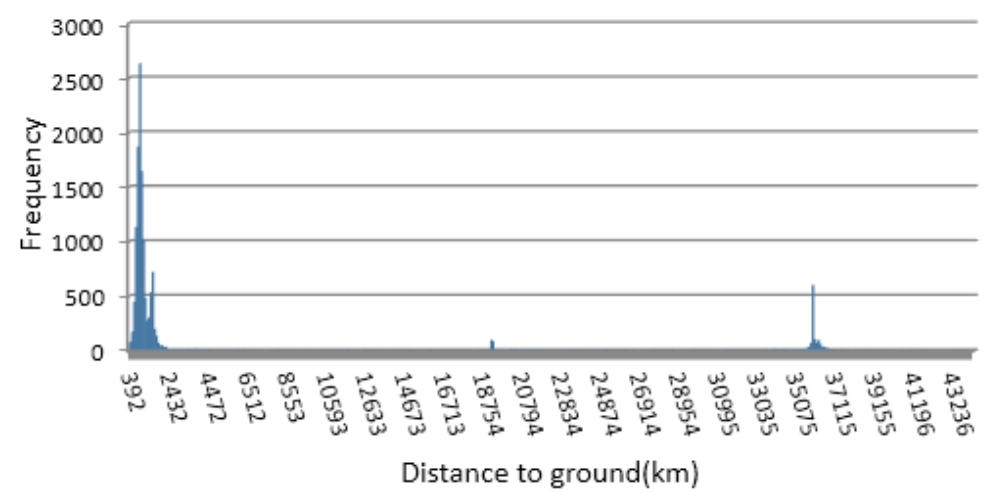

Figure 3.The Frequency Distribution Histogram of Total Space Debris

We observe that the frequency distribution of space debris in Leo appears two peaks. Comparing a variety of distribution functions, we adopt Weibull distribution function to establish the model for the distribution of the LEO Orbit with space debris, the functions are shown as follows:

$$
\begin{gathered}
F(h)=1-\exp \left[-\left(\frac{h-t_{0}}{T-t_{0}}\right)^{b}\right] \\
f(h)=\frac{b}{T-t_{0}}\left(\frac{h-t_{0}}{T-t_{0}}\right)^{b-1} \exp \left[-\left(\frac{h-t_{0}}{T-t_{0}}\right)^{b}\right]
\end{gathered}
$$

Hence, we apply the method of parameter optimization in solving double mixed Weibull distribution model. Thus far, we finally draw the probability density function and the cumulative distribution function of the space debris in LEO, and the results are shown as follows:
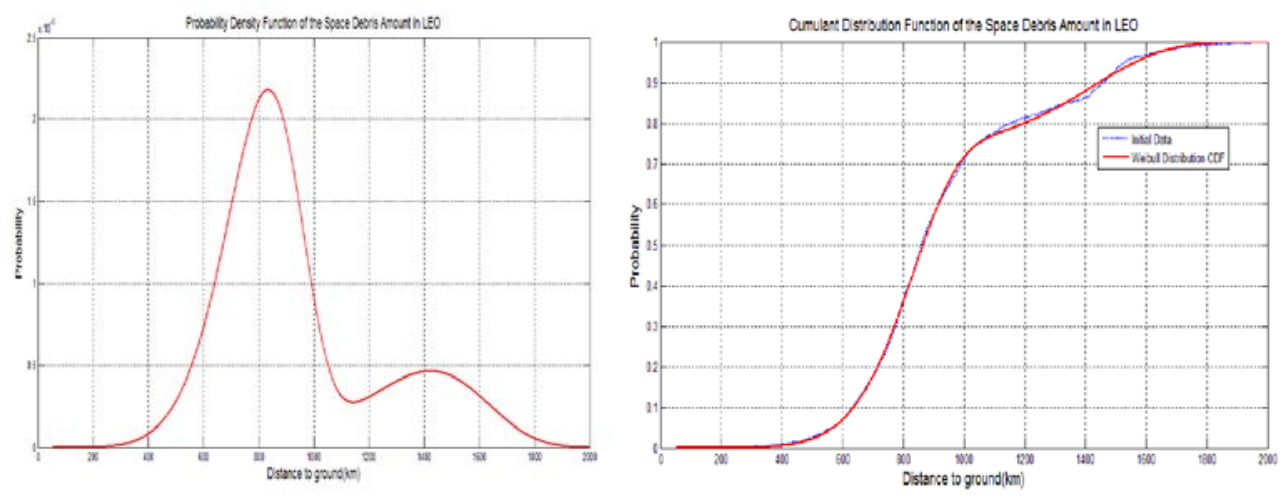

Figure 4.The PDE and CDF of the Space Debris Amount in LEO

\section{Advanced model}

\section{Optimization objective}

This thesis is to determine the best alternative for a private firm to address the space debris problem, so we should be concerned about the risks, benefits and cost of the alternative to clean space debris for private firm.

Taking costs, risks and benefits into consideration, we develop optimization model to establish the best clean-up strategy at this moment. For costs, there exist differences among different methods to remove space debris, so we define a parameter $\omega_{i}$ to measure the cost of the strategy. For benefits, we maximize the benefit by removing space debris selectively. So we define a parameter $\sigma_{i}$ to determine whether the debris is need to remove or not. For risks, we measure it by defining a new parameter $f$, which presents the possibility of removing debris successfully. These factors are combined as optimization objective, which are shown as follows: 


$$
\begin{aligned}
& \min =\sum_{i=1}^{N} \sigma_{i} w_{i} h_{i}\left(f_{\max }-f_{i}\right) \\
& \left\{\begin{array}{l}
\sigma_{i}=\left\{\begin{array}{l}
1 \quad \text { which is selected to remove } \\
0 \quad \text { which is not selected to remove }
\end{array}\right. \\
w_{i}=\left\{\begin{array}{l}
1 \quad \text { the cost of cleanup for small debris } \\
10 \quad \text { the cost of cleanup for large debris }
\end{array}\right. \\
\sum_{i=1}^{N} \sigma_{i} m_{i}{ }_{\Delta} M \\
\sum_{i=1}^{N} \sigma_{i} f_{i} \geq{ }_{\Delta} F
\end{array}\right.
\end{aligned}
$$

\section{Solving the advanced model}

Simulated Annealing algorithm is used to solve the advanced model. In this way, we obtain the best clean-up strategy, consisted of the time when to clean, the debris which to remove and the method which to choose.

After solving the objective optimization model by simulating, we find that we are proposed to carry out cleanup in 2020. Meanwhile, we determine the certain space debris that are proposed to remove and the certain clean-up strategy is shown in Figure 5.

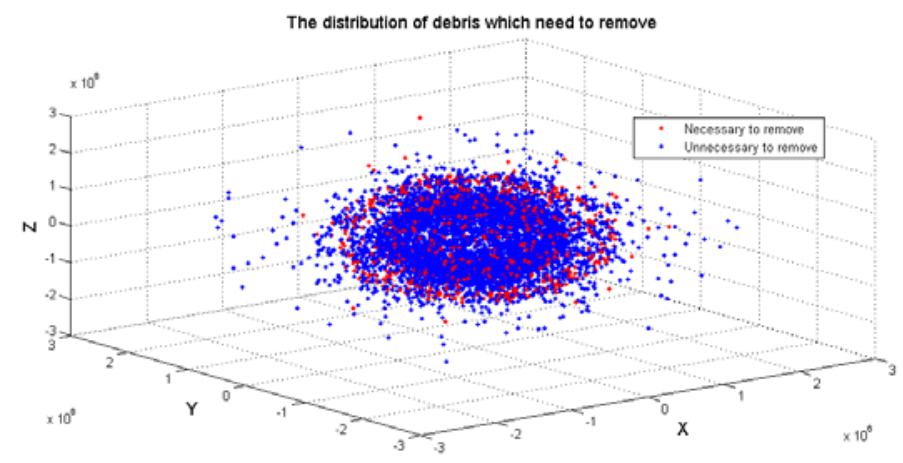

Figure 5. The Distribution of Debris that are proposed to remove Here, the red mottle presents the debris that are proposed to remove.

\section{Reference}

[1] LIU Lin, YANG Jian, WANG Jianhua, JResearch on Space Debris Mitigation Strategy in LEO, Journal of Academy of Equipment, Volume 24, 2013

[2] GONG Songbo, Research on the Establishment and Application of Space Debris Evolution Model, Harbin Institute of Technology, 2005

[3] http://jyoder.com/

[4] Liang Shuang, Mo Zhongxi, Solving the multiple sequence alignment problem based on simulated annealing algorithm, Wuhan University Journal of Natural Sciences,June 2002, Volume 7, Issue 2, pp 144-144 